\title{
Ley que prohíba el uso de celulares durante la conducción de motos para reducir accidentes
}

\author{
Law that prohibits the use of cell phones while driving motorcycles to reduce accidents \\ Lei que proíbe o uso de telefones celulares durante a condução de motocicletas para \\ reduzir acidentes
}

Artículo recibido en julio 2019

Arbitrado en agosto 2019

Publicado en octubre 2019

\section{Vladimir Quiñonez}

vladimir.quiñonez@yahoo.com ORCID: 0000-0002-6472-357X

\section{Universidad Privada "Domingo Savio" Bolivia}

RESUMEN

ABSTRACT

RESUMO

El uso de celulares mientras se conduce un vehículo motorizado trae como consecuencia accidentes de tránsito, y la ausencia de leyes que regulen esta falta, genera que incrementen, así que, la investigación se centró en una propuesta de anteproyecto de Ley Municipal que prohíba el uso de teléfonos celulares durante la conducción de vehículos motorizados para reducir los accidentes de tránsito. La metodología fue del tipo descriptiva, bajo un enfoque cuali-cuantitativo, con un diseño documental de campo, los métodos usados fueron análisis y síntesis, histórico y lógico, la técnica e instrumentos usado fue la entrevista (guía), encuesta (cuestionario) y observación directa e indirecta (guía), la población y muestra estuvo conformada por la población de la ciudad de Potosí (100) y la del parque automotor público y particular (55). Se obtuvo como resultado la creación de la ley, la cual permitirá una seguridad vial para peatones y motorizados de Bolivia.

Palabras clave: Accidente; tránsito; derecho público; derecho administrativo; derecho municipal; derecho comparado, ley de tránsito

The use of cell phones while driving a motor vehicle results in traffic accidents, and the absence of laws that regulate this lack, causes them to increase, so the investigation focused on a proposal for a preliminary draft of the Municipal Law that prohibits the use of cell phones while driving motor vehicles to reduce traffic accidents. The methodology was descriptive, under a qualitative-quantitative approach, with a field documentary design, the methods used were analysis and synthesis, historical and logical, the technique and instruments used was the interview (guide), survey (questionnaire) and direct and indirect observation (guide), the population and sample were made up of the population of the city of Potosi (100) and that of the public and private vehicle fleet (55). The result was the creation of the law, which will allow road safety for pedestrians and motorized vehicles in Bolivia.

Key words: Traffic; accident; public Law; administrative law; municipal law; comparative law, traffic law

O uso de celular na direção de veículos automotores acarreta acidentes de trânsito e a ausência de leis que regulem essa carência, faz com que aumentem, de modo que a investigação se concentrou em uma proposta de anteprojeto de Lei Municipal que proíbe o uso de telefones celulares ao dirigir veículos motorizados para reduzir acidentes de trânsito. A metodologia foi descritiva, com abordagem qualitativo-quantitativa, com delineamento documental de campo, os métodos utilizados foram análise e síntese, histórico e lógico, a técnica e os instrumentos utilizados foram a entrevista (guia), enquete (questionário) e observação direta e indireta (guia), a população e a amostra foram constituídas pela população da cidade de Potosí (100) e pela frota de veículos públicos e privados (55). O resultado foi a criação da lei, que vai permitir a segurança viária de pedestres e veículos motorizados na Bolívia.

Palavras-chave: Acidente; trânsito; lei pública; direito Administrativo; lei municipal; direito comparado, direito de trânsito 


\section{INTRODUCCIÓN}

El siglo XXI ha traído consigo una nueva forma de comunicación personal y grupal para una sociedad, la telefonía móvil, en donde mediante una infraestructura y un mercado de bienes y servicios, se ha logrado desarrollar un nuevo enfoque que afecta la cultura, la economía, la política, la geografía y en general a la sociedad.

Mediante esta nueva forma de comunicación se mantienen relaciones sociales que involucran el sentido comercial, a través de un mercado de bienes y servicios; en donde existe una necesidad que demanda un modelo y una serie de mecanismos para su satisfacción por medio de su utilización, generando nuevas percepciones de vida y cambios a nivel general.

La expansión acelerada del mercado de telefonía móvil a nivel mundial es uno de los fenómenos más destacados en el campo de la comunicación en la última década. Sus implicaciones son diversas, pues el teléfono celular afecta no sólo el desarrollo de estructuras sociales y actividades económicas sino que cambia la naturaleza misma de la comunicación y de las relaciones sociales modificando también la manera como se construye la identidad y se conceptualiza el espacio su movilidad, conectividad e instantaneidad lo han convertido en la tecnología de comunicación personal más usada alrededor del mundo y en uno de los objeto de consumo más deseados por grandes segmentos de población.

La telefonía móvil es un servicio que ha experimentado un crecimiento exponencial en años recientes, y en la actualidad la demanda no da señales de estabilizarse. Son distintos los factores que explican el importante crecimiento de ésta, ya que esto implica mejoras en la comunicación, atención de nuevos sistemas operativos, numerosas aplicaciones, fácil acceso a internet, entre otros beneficios que ofrece, cada vez más actualizados.

En la actualidad la telefonía celular es un medio de comunicación de uso masivo y sorprende ver las propuestas de las compañías en las que a tecnología celular respecta. En nuestro realidad presente cada día son más los usuarios que se suman a las compañías de telefonía móvil, cabe destacar que gran cantidad de estos usuarios son jóvenes que adquieren equipos de última generación; puesto que gran parte de estos equipos cuentan con acceso a Internet y la tecnología bluetooth e infrarrojo por medio del cual se pueden descargar y transferir datos. (GEOSALUD, 2012).

Por otro lado, los accidentes automovilísticos son una de las problemáticas más comunes en la actualidad ya que los vehículos son uno de los medios de transporte más utilizados en estos tiempos los cuales son indispensables para trasladarse de un lugar a otro. Pero la imprudencia de las personas al realizar el uso del celular durante la conducción ha originado varios accidentes de tránsito. 
Cabe destacar, que la Organización Mundial de la Salud (OMS), reporta que cada año los accidentes de tránsito causan la muerte de aproximadamente 1,3 millones de personas en todo el mundo. De no aplicar medidas para evitarlo, se prevé que para el año 2020 los accidentes de tránsito causarán 1,9 millones de muertes anuales. Tomando en cuenta este panorama, sería necesario cuestionarse acerca de los accidentes que pueden derivarse ante el uso desmedido del celular.

De esta forma la OMS (2011) señala que:

Estudios realizados determinaron que conducir mientras se usa el teléfono celular reduce la actividad cerebral en un $37 \%$, puesto que cuando se intentan hacer dos cosas a la vez, una de las dos se ve afectada. De este modo, la realización de otra tarea cognitiva durante la conducción de un carro degrada sustancialmente el rendimiento de la persona que conduce. Aunado a esto, se ha notado un aumento en los últimos años la existencia de una actitud compulsiva de revisar el celular en cualquier momento, de tal manera que en investigaciones realizadas, $51 \%$ de los usuarios asegura que sería muy difícil renunciar a sus teléfonos celulares.(s/p).

Por ende, se asume que los accidentes de tránsito por el uso del celular durante la conducción es uno de los accidentes de tránsito que va en constante crecimiento ocasionado peligro tanto para peatones como para los conductores de los vehículos motorizados.

Cabe destacar, que en Perú, Chile, Argentina, República, Dominicana, España, Australia, en las leyes de seguridad vial, el hablar por teléfono y a la vez conducir un vehículo es considerado como infracción, garantizando así la seguridad del peatón y la del conductor, estas faltas anteriormente mencionadas cometidas por los conductores de vehículos son sancionadas, ya que esta medida se contribuye a una conducta peligrosa al volante.

Si embargo, en Bolivia, el código de tránsito está encargada de regular las sanciones e infracciones originadas por los vehículos automotores, pero esta norma fue elaborada hace 37 años atrás, por lo tanto, este código deja de ser efectivo a los avances tecnológicos, los cuales, son causantes de accidentes de tránsito, dejando así los nuevos tipos de infracciones sin una sanción, tal es el caso del uso del celular durante la conducción del vehículo motorizado.

Si bien esta norma es general en todo el territorio nacional, cada municipio tiene la facultad de elaborar leyes municipales acorde a sus necesidades, por lo tanto, la investigación se centra en las facultades del municipio como elaborador de leyes municipales, en este caso una ley de prohibición del uso del celular durante la conducción de un vehículo motorizado, teniendo como referencia la elevación de los accidentes de tránsito dentro del municipio de Potosí, ya que sin ninguna ley que 
regule esta falta cometida por los conductores de movilidades, este tipo de accidentes se incrementarían, ocasionando así una inseguridad vial en el municipio anteriormente mencionado. Así que, el objetivo central de la investigación se basa en proponer un anteproyecto de Ley Municipal que prohíba el uso de teléfonos celulares durante la conducción de vehículos motorizados para reducir los accidentes de tránsito en la Ciudad de Potosí.

Con la investigación se pretende dar un aporte importante para Bolivia, ya que estará orientado a ofrecer un modelo jurídico, cuyo objetivo es disminuir el nivel de accidentes causados por el uso del móvil en el tiempo de la conducción de un motorizado en la ciudad de Potosí, mediante la aprobación de una Ley Municipal que prohíba el uso del teléfono celular durante la conducción de un vehículo motorizado, dando lugar al cumplimiento de las disposiciones legales emanadas por el Estado Plurinacional, que otorga competencias exclusivas a los Gobiernos Autónomos Municipales.

\section{Marco Teórico}

\section{Derecho Público}

Osorio (2002), define al Derecho Público como:

la parte del ordenamiento jurídico que regula las relaciones entre las personas o entidades privadas con los órganos que ostentan el poder público cuando estos últimos actúan en ejercicio de sus legítimas potestades públicas (jurisdiccionales, administrativas, según la naturaleza del órgano que las ejerce) y de acuerdo con el procedimiento legalmente establecido, y de los órganos de la Administración pública entre sí.

Además, Máynez (1944) define Derecho Público como: "Las relaciones entre el Estado y los particulares, o entre entes estatales entre sí" (s/p).

Por otro lado, Cabanellas (2006), lo define como: "El que regla los actos de las personas cuando se desenvuelven dentro del interés general que tiene por fin el Estado, en virtud de delegación directa o mediata del poder público"(s/p).

Así que, se indica que el Derecho Público se rige en un ordenamiento jurídico siendo que es la integración de las normas en un conjunto o sistema de normas e instituciones organizado y coherente, de manera que éstas adquieren relevancia por la posición que ocupan en el mismo, puede decirse que todo ordenamiento jurídico tiene su propio sistema o coherencia lógica de valores. 


\section{Derecho Administrativo}

Para Cabanellas (2006), el Derecho Administrativo es: "El conjunto de normas reguladoras de las instituciones sociales y de los actos del Poder ejecutivo para la realización de los fines de publica utilidad" (s/p).

Otra definición señala que Derecho Administrativo es: "Un Conjunto de normas que regulan esencialmente el ejercicio de la función administrativa, es decir, de un lado, la organización del Poder Ejecutivo y el ejercicio efectivo de la función misma y las relaciones a que da lugar este ejercicio". (Oyanguren, 1991)

De esta manera, se indica que el Derecho Administrativo es un conjunto de normas por lo que es una regla dirigida a la ordenación del comportamiento humano prescrita por una autoridad en este caso el Poder Ejecutivo que es aquel que se encarga de las funciones políticas y administrativas de un Estado.

\section{Derecho Municipal}

Osorio (2002), define al Derecho Municipal como "La rama del Derecho Público institucional con acción pública, que estudia los problemas políticos jurídicos y sociales del urbanismo" (s/p).

Sin embargo, Bielsa (1938), indica que: "El derecho municipal es inexistente como una rama autónoma considerándolo simplemente como el conjunto de preceptos o principios del Derecho Administrativo general aplicables a la esfera comunal" (s/p).

Por consiguiente, el derecho municipal deriva del Derecho Público el cual estudia el actuar social jurídico y político de principios, técnicas y prácticas y cuya aplicación permite establecer sistemas racionales de esfuerzo cooperativo, a través de los cuales se puede alcanzar propósitos comunes que beneficie a un grupo social, en el ámbito técnico-administrativas y financieras que regular las municipalidades y el desenvolvimiento del municipio.

\section{Ley Municipal}

Osorio (2002), señala que la Ley Municipal:

Es aquella norma general y de carácter obligatorio que ha sido dictaminado por el consejo municipal con el objetivo de establecer relaciones entre municipio y persona con el objetivo de regular el orden municipal. En caso de que las leyes no sean cumplidas, la fuerza pública tiene el deber y obligación de sancionar a la persona o institución correspondiente. $(\mathrm{s} / \mathrm{p})$. 
Otra definición indica que:

La ley municipal es una norma jurídica dictada por una autoridad municipal competente, previo debate de los alcances y el texto que impulsa la misma y que deberá observar un cumplimiento obligatorio por parte de todos los ciudadanos, sin excepción, de un Municipio. (Cabanellas, 2006)

De los conceptos anteriores se refiere al derecho municipal como una norma que regla las conductas que son impuestas por una autoridad jerárquica que este a su vez establece su alcance y su cumplimiento.

\section{Seguridad vial}

Para Osorio (2002),

La seguridad vial es la prevención de accidentes de tránsito o la minimización de sus efectos, especialmente para la vida y la salud de las personas, cuando tuviera lugar un hecho no deseado de tránsito. También se refiere a las tecnologías empleadas para dicho fin en cualquier medio de desplazamiento terrestre. ( $\mathrm{s} / \mathrm{p}$ ).

Por otro lado, Cabanellas (2006), señala que:

La seguridad vial es el conjunto de acciones y mecanismos que garantizan el buen funcionamiento de la circulación del tránsito, a través del conocimiento y cumplimiento de las leyes y reglamentos, bien sea como conductor, peatón o pasajero, para usar correctamente las vías públicas y previniendo los accidentes de tránsito.(s/p)

De esta forma, se puede decir que la seguridad de un estado en el cual los peligros y las condiciones que pueden provocar daños de tipo físico, psicológico o material son controlados para preservar la salud y el bienestar de las personas, conductores, vinculadas a lugares por los cuales se transita ya sea a pie o en vehículos. Por lo tanto, la seguridad vial es considerado como la prevención de accidentes de tráfico con el objetivo de proteger la vida de las personas y los conductores de vehículos para un correcto ordenamiento vial.

\section{Derecho Comparado}

Chile: artículo 200. Indica que son infracciones graves la siguiente: Numeral 32. Conducir haciendo uso de un teléfono celular u otro aparato de telecomunicaciones, 
salvo que tal uso se efectúe por medio de un sistema de "manos libres", cuyas características serán determinadas por reglamento.

Argentina: artículo 2: Se modifica el artículo 77 de la Ley de Tránsito N²4449, incorporando un nuevo inciso que establece como falta grave: Hablar por teléfono durante la conducción de vehículos motorizados y la instalación de pantallas de DVD. en la parte delantera de vehículos de todo tipo.

\section{MÉTODO}

La metodología de la investigación fue del tipo descriptivo. Se asumió esta posición en razón, de que se realizó un registro detallado del comportamiento específico de las dos variables identificadas: Proyecto de Ley Municipal (variable independiente), y los accidentes de tránsito por el uso de teléfonos celular durante la conducción de vehículos (variable dependiente).

A su vez, el diseño de la investigación en correspondencia con el problema planteado y atendiendo al objetivo general propuesto, fue de tipo documental y de campo. La primera de ella por cuanto se realizó una investigación documental del problema de investigación. La segunda derivo de la necesidad de investigar la situación actual de los accidentes de tránsito ocasionados por el uso del celular durante la conducción, a través de los instrumentos de recolección que fueron empleados en el diagnóstico de la investigación.

El enfoque de investigación que se asumió fue cuanti-cualitativo, en razón que la investigación determinó un acercamiento al problema a partir de elementos cuantificables y calificables, para establecer un diagnóstico real del mismo, de manera que a partir de los resultados se pudo plantear una propuesta de solución.

Los métodos que se aplicaron fueron: análisis-síntesis, con la finalidad de estructurar los componentes de la Ley Municipal, se utilizó el estudio la literatura consultada y en la interpretación de los datos empíricos. Así como para arribar a conclusiones a partir de fuentes bibliográficas y sistematizar las características de la propuesta referidos al servicio público de taxis. Histórico-lógico, porque se estudió documentos existentes como bibliografías, teorías, derecho comparado, Leyes y otras disposiciones legales referidas al uso del celular durante la conducción de un vehículo. Inducción-deducción, este método se utilizó con el fin de encontrar diferencias y semejanzas de teorías existentes referidas al uso del celular durante la conducción de un vehículo.

Por otro lado, las técnicas e instrumentos, utilizados para el desarrollo de la investigación fueron: análisis documental, entrevista, encuesta, y observación directa, abierta, no participante y externa.

Estudio Documental. Con la finalidad de recopilar y registrar fundamentos documentales relacionados con la investigación, se estudiaron los accidentes de 
tránsito por la distracción que origina el celular, registradas en la Unidad Operativo de Transito. El instrumento utilizado fue la revisión documental, aplicado en el diagnóstico con el fin de conocer las denuncias registradas de los accidentes de tránsito por el uso del celular durante la conducción de un vehículo.

Entrevista. Este instrumento fue aplicado, al Presidente Consejo Municipal del Transporte, Director de la Unidad Operativa de Transito con la finalidad diagnóstica de conocer su percepción y profundizar el tema de investigación. El instrumento utilizado fue la guía de entrevista, aplicada en el diagnóstico.

Encuesta. Este instrumento fue aplicado a personas mayores de edad comprendidas entre los 18 a 50 años y conductores de vehículos motorizados del de la cuidad de Potosí, con la finalidad de conocer percepciones a cerca de los accidentes de tránsito por el uso del celular durante la conducción de un vehículo.

El instrumento utilizado fue el cuestionario, aplicado en el diagnóstico.

Observación directa, abierta, no participante y externa. Este instrumento fue aplicado con la finalidad de verificar el grado de incremento de distracción por el uso del celular durante la conducción, así como el comportamiento de los sujetos actores de esta modalidad de transporte. El instrumento utilizado fue la guía de observación.

A demás, la población y muestra utilizada en la investigación estuvo conformada por la población de la ciudad de Potosí, según el CENSO de 2013 fue de 94387 personas comprendidas entre 18 a 50 años. Por lo que, la muestra usada en la investigación fue de 100 personas. Por otro lado, también se usó la población conformada por el parque automotor público y particular, tomando como base el número referido por el INE de la ciudad Potosí, con un total de 50936 movilidades, según el informe brindado, de la gestión 2014. De esta forma la muestra que se usó para la investigación fue de 55 conductores de vehículos privados y públicos en la ciudad de Potosí.

\section{RESULTADOS Y DISCUSIÓN}

\section{Incremento constante de los accidentes de tránsito}

Tabla 1. Accidentes de tránsito ocurrido por el uso del celular durante la conducción vehicular.

\begin{tabular}{ccc}
\hline ALTERNATIVAS & FRECUENCIA & $\%$ \\
\hline $\mathrm{Si}$ & 93 & 93 \\
$\mathrm{No}$ & 7 & 7 \\
\hline TOTAL & $\mathbf{1 0 0}$ & $\mathbf{1 0 0}$ \\
\hline
\end{tabular}


Como se muestra en la tabla 1, de 100 personas encuestadas el 93\%, tiene conocimiento sobre la existencia de accidentes de tránsito ocasionados por el uso de teléfonos celulares durante la conducción de vehículos, y un 7\%, desconoce esta situación.

De los datos que se obtuvo en el cuestionario, se pudo establecer sin lugar a dudas que los accidentes de tránsito por el uso de celular se han convertido en un mal hábito por parte de los conductores, que estos a su vez ocasiona prejuicios para la sociedad de un municipio, si se toma en cuenta las cifras señaladas en la tabla se observa que las personas conocen sobre el incremento de los accidentes de tránsito a causa del uso del celular durante la conducción, por lo tanto no es un tema en reserva o que no llegue a afectar a un municipio.

De la misma manera se realizó encuestas a los conductores de servicio público como particulares.

Se aprecia en la tabla 2 que de los 55 conductores de movilidades particulares y públicos el $11 \%$, no conoce, sobre accidentes de tránsito a causa del uso del celular y un $89 \%$, de los encuestados conoce sobre este tipo de accidentes en la ciudad de Potosí.

Realizando un análisis de los datos obtenidos en la encuesta se señaló que el incremento de accidentes de tránsito a causa del uso del celular es cada vez más frecuente, debido a la distracción que provoca el mismo en el momento de la conducción de un vehículo, el otro porcentaje de los conductores que desconoce la existencia de este tipo de accidentes, se justifica por la falta de información a través de los medios de comunicación.

Tabla 2. Accidentes de tránsito ocurrido por el uso del celular durante la conducción vehicular (conductores).

\begin{tabular}{ccc}
\hline ALTERNATIVAS & FRECUENCIA & \% \\
\hline $\mathrm{Si}$ & 49 & 89 \\
No & 6 & 11 \\
\hline TOTAL & $\mathbf{5 5}$ & $\mathbf{1 0 0}$
\end{tabular}




\section{Norma que regule y/o sancione el uso del celular durante la conducción de un} vehículo motorizado

Tabla 3. Considera que una Norma Municipal contribuiría al tránsito a efectiviza el control del uso de teléfonos celulares durante la conducción de vehículos

\begin{tabular}{ccc}
\hline ALTERNATIVAS & FRECUENCIA & $\%$ \\
\hline $\mathrm{Si}$ & 97 & 97 \\
$\mathrm{No}$ & 3 & 3 \\
\hline TOTAL & $\mathbf{1 0 0}$ & $\mathbf{1 0 0}$ \\
\hline
\end{tabular}

Como se puede observar en la tabla 3, de 100 personas encuestadas el $97 \%$, de las personas señalan que una norma municipal contribuiría al tránsito a efectivizar el control del uso de teléfonos celulares durante la conducción de vehículos y solo un $3 \%$, no están de acuerdo.

De esta forma, se señaló que la elaboración de una Ley Municipal estaría conforme a la solicitud de la sociedad de un municipio para reducir y prevenir los accidentes de tránsito por el uso del teléfono celular durante la conducción de un vehículo lo cual ayudaría y mejoraría la seguridad vial del municipio de Potosí.

\section{La poca concentración durante la conducción del vehículo motorizado.}

En la tabla 4, se muestra que los 55 conductores de movilidades particulares y públicos asumen un $77 \%$ que si utilizan el celular durante la conducción de su vehículo y solo un $23 \%$ asume que no realiza la utilización del dispositivo móvil.

Tabla 4. Uso del celular durante la conducción del vehículo ( conductores).

\begin{tabular}{ccc}
\hline ALTERNATIVAS & FRECUENCIA & $\%$ \\
\hline Si & 42 & 77 \\
No & 13 & 23 \\
\hline TOTAL & 55 & 100 \\
\hline
\end{tabular}

De esta forma, se dedujo que es un peligro constante y que va en crecimiento el uso del celular durante la conducción de un vehículo, el efecto de esta situación, es que los conductores realicen de manera consiente su labor de conducir una movilidad con toda la seguridad y fuera de distracción. 


\section{Inseguridad vial}

Como se observa en la tabla 5, de 100 personas encuestadas el 53\%, han sido testigos, y un $47 \%$, no han sido testigos de accidentes de tránsito ocasionados por el uso de teléfonos celulares durante la conducción de vehículos.

Tabla 5. Ha sido testigo de algunos accidentes de tránsito ocasionados por el uso de teléfonos celulares.

\begin{tabular}{ccc}
\hline ALTERNATIVAS & FRECUENCIA & $\%$ \\
\hline Si & 53 & 53 \\
No & 47 & 47 \\
\hline TOTAL & 100 & 100 \\
\hline
\end{tabular}

Tomando en cuenta los datos obtenidos se interpretó, que si bien existe casi un igual de porcentajes entre aquellos que fueron testigos y quienes no, de accidentes de tránsito ocasionados por el uso de teléfonos celulares durante la conducción de vehículos, se toma en cuenta que una mayoría si pudieron apreciar este tipo de accidentes, haciendo relevancia que casi de manera continua se viene sucintado este tipo de hechos de tránsito donde se tiene como perjudicados a aquellos que lo cometen y a aquellos que lo sufren teniendo así una inseguridad vial que cotidianamente está en crecimiento

\section{Mal hábito del uso del celular}

Para este indicador se utilizó dos instrumentos siendo como primer instrumento la entrevista al Coronel Guillermo Cejas. Se obtuvo como respuestas, que sí, es un mal hábito que tienen los conductores de movilidades ya que por normas los conductores conocen que cuando el celular suena, para contestar deberían parquear la movilidad, poner luces de parque y estacionarse en un lugar cómodo y luego hablar el tiempo que desee por su celular, de esta manera, no causaría la inseguridad al tránsito del peatón o a otras movilidades.

El segundo instrumento que se utilizó fuer la observación directa en las calles de la ciudad de Potosí con los siguientes resultados. Uso del teléfono celular. La observación se realizó en las calles de la ciudad de Potosí por el lapso de 1h: 30 min:

- Calle Bustillo Bolívar de 100 movilidades se detectó a 68 conductores haciendo el uso del teléfono celular de los cuales a 21 conductores se apreció en una desconcentración total a la conducción de su movilidad. 
- Calle Oruro Bolívar de 100 movilidades se detectó a 53 conductores haciendo el uso del teléfono celular, de los cuales 15 conductores brindaron una inseguridad vial a los peatones.

- Avenida Universitaria Altura pasarela 100 movilidades se detectó a 56 conductores haciendo el uso del teléfono celular, donde todos ellos realizaban manejo inapropiado del celular

Cabe destacar, que la inseguridad vial se reflejó en la observación directa que se realizó en la investigación, ya que de 100 movilidades se apreció que 56 conductores utilizaban su teléfono celular y de estos todos 56 conductores mostraron la dependencia que tienen del celular haciendo un mal hábito en la conducción del vehículo, pero con un ordenamiento legal acorde se puede frenar a tiempo este mal hábito.

\section{Propuesta de anteproyecto de Ley Municipal de prohibición de uso de teléfonos móviles celulares durante la conducción de vehículos motorizados para reducir los accidentes de tránsito}

Con el anteproyecto de Ley Municipal de prohibición de uso de teléfonos móviles celulares durante la conducción de vehículos motorizados se busca disminuir los accidentes de tránsito en la ciudad de Potosí, por lo cual se plantea alternativas que darán soluciones a las adversidades identificadas en el diagnóstico, finalmente de lograr concretarse la propuesta, permitirá una seguridad vial para peatones y motorizados, con adecuadas medidas de prevención, control y monitoreo.

\section{Fundamentación jurídica}

La fundamentación jurídica para la creación de la ley municipal toma en cuenta varias disposiciones legales.

Constitución Política del Estado

Ley General del Transporte

Ley de Gobiernos Autónomos Municipales

Ley Marco de Autonomías y Descentralización 


\section{Ley Municipal de prohibición de uso del teléfono celular durante la conducción de un vehículo motorizado en la ciudad de Potosí}

Está conformado por un título y cuatro capítulos.

- Capitulo I. Disposiciones generales. Se desarrolla las disposiciones generales (artículo 1) y el campo de aplicación (artículo 2).

- Capítulo II. Principios y definiciones. Se desarrollan las definiciones: Accidente de tránsito, calle, calle peatonal, calzada, camino, conductor o motorista, vehículo, teléfono celular móvil (artículo 3).

- Capítulo III. De la prohibición, sanción y control del uso del teléfono celular durante la conducción de vehículo motorizado. Se desarrolla la prohibición (artículo 4), sanción (artículo 5) y control/ monitoreo ( artículo 6).

- Capitulo IV. Disposiciones finales. Vigencia de la ley desde su aprobación (artículo 7).

De esta manera, queda plasmado en el anteproyecto de ley Municipal de prohibición de uso del teléfono celular durante la conducción de un vehículo motorizado en la ciudad de Potosí, la prohibición, sanción y control/monitoreo:

Artículo 4. (Prohibición). Queda prohibido el uso de teléfonos celulares a toda persona mientras este conduciendo un vehículo automotor sea público o particular en las vías públicas.

Artículo 5. (Sanción). La persona que sea sorprendida por vez primera usando teléfonos celulares mientras este conduciendo un vehículo a motor en las vías públicas será sancionado con el pago de una multa del veinticinco por ciento (25\%) del salario mínimo. A la reincidencia de la falta será sancionado con el pago de una multa del cincuenta por ciento (50\%) del salario mínimo. A la tercera vez de incumplir con la presente ley se le sancionara con la retención de la licencia de conducir.

Artículo 6. (Control y Monitoreo) La Unidad Operativa de Tránsito se encarga del control y monitoreo para darle estricto cumplimiento a la nueva ley.

\section{CONCLUSIONES}

Partiendo del objetivo central de la investigación, se puede decir que se cumplió con dicho objetivo, por lo tanto, se ha podido establecer que es una necesidad primordial la creación de la ley municipal que prohíba el uso de teléfonos celulares durante la conducción de un vehículo motorizado siendo dado a que el código de tránsito del municipio, está quedando obsoleto a las nuevas tecnologías, por lo que 
si bien por las autonomías regionales nos faculta a crear leyes en beneficio de la sociedad, en este caso en beneficio de la seguridad vial del municipio de Potosí.

Para finalizar, queda de parte del Estado a través los Gobiernos Autónomos Municipales, aprobar políticas dirigidas al sector de transporte urbano municipal, para así poder garantizar a los conductores como a los peatones un sistema de seguridad vial que proteja y garantice su estabilidad vial.

\section{REFERENCÍAS}

Argentina, R. D. (2014). Ley De Transito 24449. Buenos Aires

Bielsa, R. (1938). Derecho Administrativo. Buenos Aires: Taurus

Cabanellas, T. (2006). Diccionario Jurídico Elemental. Healista

Chile, R. D. (2005). Ley De Transito 18920. Santiago Geosalud. (2012). Recuperado el 14 de enero de 2015, de www.geosalud.com
Máynez, G. (1944). Introducción al estudio del Derecho. Instituto de estudio político. México

OMS (2011). Uso del celular al volante. Bélgica: Laussane

Osorio, M. (2002). Diccionario de Ciencias Jurídicas Políticas y Sociales. Buenos Aires: Heliasta

Oyanguren, A. (1991). Manual Elemental De Derecho Administrativo. Universidad Nacional Autónoma de Nicaragua, León 